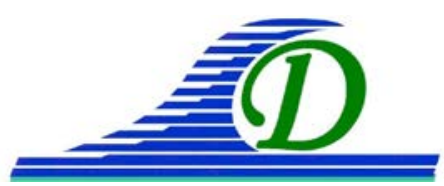

XIII İ̀es Journées Nationales Génie Côtier - Génie Civil

Dunkerque, 2-4 juillet 2014

DOI:10.5150/jngcgc.2014.059 @ Editions Paralia CFL

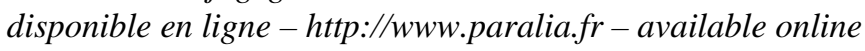

\title{
Approche géophysique de la couverture sédimentaire au large des Pays de Monts
}

\author{
Agnès BALTZER ${ }^{1}$, Mohamed MAANAN ${ }^{1}$, Nicolas ROLLO ${ }^{1}$, Aurélie DURAND ${ }^{1}$ \\ 1. Université de Nantes, Laboratoire Géolittomer/IGARUN, UMR 6554, \\ Chemin de la Censive du Tertre, BP 81227, 44312 Nantes Cédex 3, France. \\ agnes.baltzer1@univ-nantes.fr
}

\section{Résumé :}

Sur la côte Ouest de la Vendée, une prospection sismique a été réalisée le long du littoral entre St Gilles Croix de vie et Notre Dame de Monts. Cinquante-huit profils très haute résolution ont été ainsi acquis à l'aide du Boomer Seistec IKB de l’Université de Caen. L'objectif principal de cette étude est d'évaluer et de cartographier le stock sédimentaire disponible en domaine marin. Le second objectif est d'essayer de comprendre la nature et la provenance de ces sédiments afin de savoir si cette couverture sédimentaire est susceptible de recharger les plages de cette portion du littoral vendéen. Des hauteurs extrêmes supérieures à $9 \mathrm{~m}$ de houles venant de l'Ouest sont régulièrement enregistrées au large de l’Ile d'Yeu, confirmant l'existence d'un environnement particulièrement dynamique. La configuration morphologique particulière de cette Ile, prolongée par le pont d'Yeu, structure synclinale reliant l'Ile au continent, agit alors comme un obstacle à la propagation de cette houle d'Ouest, permettant de préserver les dépôts de sédiments potentiels.

Mots-clés : Vendée, Sédimentologie, Sable, Géophysique, Prisme côtier.

\section{Introduction}

Les dégâts engendrés par la tempête Xynthia ont mis en lumière une double déficience au niveau du littoral régional (i) une déficience de certaines défenses côtières artificielles -mal adaptées et/ou fragilisés (digue)- et en certains endroits naturelles (cordon dunaire avec brèche correspondant à une disparition du service protection de ces édifices naturels) et (ii) une déficience en matière de connaissance fine de la topographie -risque potentiel ou réel de submersion- que le dispositif Litto3D devrait combler dans sa partie terrestre. Cette recherche s'inscrit dans le cadre de l'étude des interactions nature/société dans la zone côtière et dans le contexte plus global de l'érosion côtière. Elle a pour ambition de replacer l'étude de l'évolution des plages dans la communauté de communes des Pays de Monts dans un contexte physique (élévation du niveau de la mer et redistribution des stocks de sables disponibles depuis la fin de l'Holocène, modification des forçages météo-marins sur un court terme, raréfaction constatée du sable amenant à un déséquilibre accentué de la dynamique du couple plage/dune). 
Ainsi l'étude de reconnaissance des budgets sédimentaires dans les petits fonds s'intègre aux travaux réalisés dans le cadre de la création de l'Observatoire du Littoral des Pays de Monts (OLPM) en partenariat avec le BRGM, l'ONF, la Communauté de communes Océan-Marais de Monts et l'Institut de Géographie et d'Aménagement Régional de l'Université de Nantes (IGARUN). Une reconnaissance géophysique sur la côte vendéenne a été réalisée en mai 2012 (figure 1), afin de cartographier les stocks sédimentaires disponibles susceptibles de pouvoir recharger les plages du littoral des pays de Monts. L'objectif est d'appréhender les processus morpho-sédimentaires de secteurs qui seraient en érosion et/ou à risque de submersion marine.

Man de pasitica des profils sismiques Hoomer Seistes

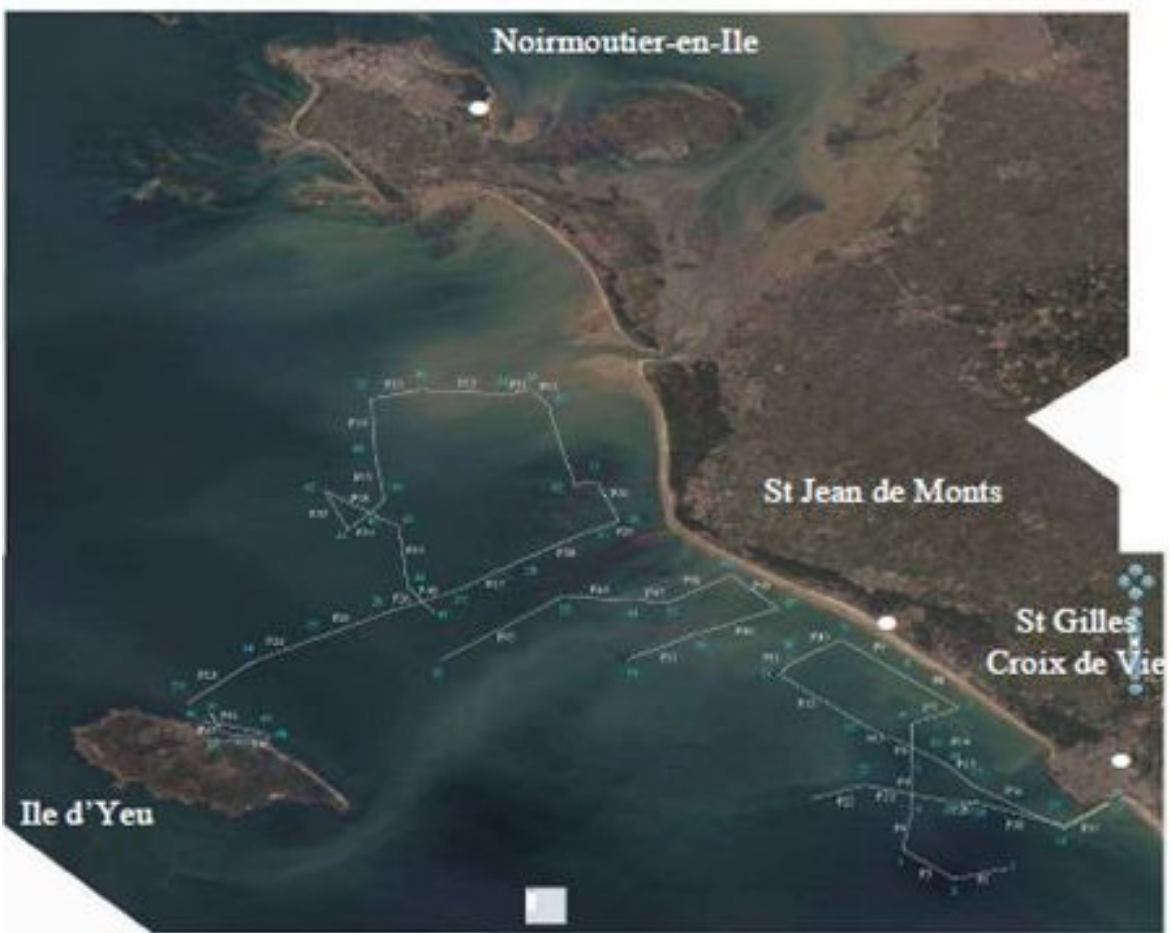

Figure 1. Localisation des différents profils sismiques réalisés en mai 2012.

\section{Cadre géologique}

Cette portion du littoral appartient aux plateaux vendéens qui s'étendent du Plateau du Four au Pays d'Olonne. Cet ensemble tabulaire, constitué essentiellement de calcaire éocène dolomitisé (46-37 Ma), s'étend approximativement sur $100 \mathrm{~km}$ et forme l'une des surfaces rocheuses les plus vastes des côtes atlantiques françaises (VANNEY, 1977).

Au sud-est du Plateau des bœufs, le Coureau Ilais correspond à un seuil rocheux calcaire, partiellement creusé de dépressions, situé entre le socle en gneiss et granit de l'Ile d'Yeu et la côte vendéenne (figure 1). En dehors de quelques falaises de micashistes (à St Gilles Croix de Vie), le littoral et son arrière-pays immédiat 


\section{XIII ${ }^{\text {èmes }}$ Journées Nationales Génie Côtier - Génie Civil \\ Dunkerque, 2-4 juillet 2014}

témoignent d'un important remblaiement. En arrière d'un immense cordon dunaire, les anciennes dépressions des Pays de Monts furent colmatées par d'épaisses argiles flandriennes (VANNEY, 1977 ; TERS, 1961) lors de la remontée du niveau marin. Ce cordon dunaire atteint une hauteur souvent d'une dizaine de mètres mais rarement de plus de 15 à 20 mètres. Sa largeur maximale est de $2 \mathrm{~km}$ au Nord et de $3 \mathrm{~km}$ au Sud, atteignant $700 \mathrm{~m}$ au niveau du pont d'Yeu (DHI, 2007).

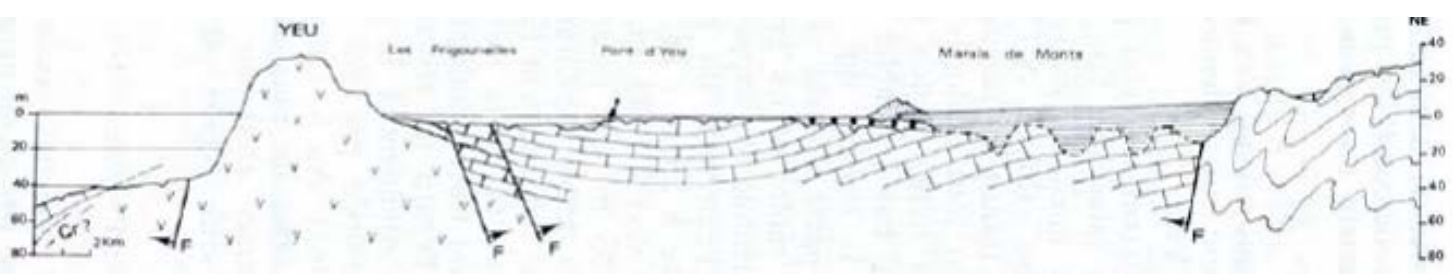

Figure 2. Coupe géologique du Coureau Ilais d'après VANNEY (1977).

\section{Outils et méthodes}

Le principe de la prospection des domaines sous-marins par géophysique repose sur l'étude des échos d'une onde acoustique émise artificiellement et réfléchie par des interfaces : le fond de la mer et les limites entre les différentes couches qui constituent les sédiments déposés sous l'eau. Lorsque les ondes émises sont de faible puissance et de très haute fréquence (quelques dizaines à quelques centaines de $\mathrm{kHz}$ ), elles ne peuvent pas pénétrer dans le sédiment ; elles sont réfléchies par le fond de la mer seulement. Plus les ondes émises sont de forte puissance et de basse fréquence (quelques dizaines de $\mathrm{Hz}$ à quelques milliers de $\mathrm{Hz}$ ), plus elles pourront pénétrer profondément dans les sédiments et restituer ainsi la géométrie interne des différentes couches qui les composent. Au contraire de la sismique dite "conventionnelle" caractérisée par une importante pénétration (quelques km pour la recherche pétrolière) et une basse résolution $(10 \mathrm{~m})$, la sismique utilisée pour étudier les domaines littoraux, où l'épaisseur des sédiments n'excède pas quelques dizaines de mètres en général, est dite "très haute résolution". Elle répond à une double nécessité : l'étude très fine des sédiments (résolution de l'ordre de $25 \mathrm{~cm}$ ) ainsi qu'une mise en oeuvre de l'outil dans des environnements peu profonds, à partir de petites embarcations.

Le Boomer Seistec (Boomer IKB - Seistec, figure 3) a été acquis par le laboratoire de Morphologie Continentale et Côtière de l'Université de Caen, dans le cadre du Pôle Géophysique Très Haute Résolution de Normandie (http://zbar.free.fr). La source d'émission de l'onde acoustique est électromécanique: une plaque (un boomer) se déforme sous l'effet d'une décharge électrique de haute tension de $4000 \mathrm{~V}$. Les échos de l'onde émise sont réceptionnés par des hydrophones placés prés de la plaque. Sa fréquence d'émission recouvre une bande de 1 à $10 \mathrm{kHz}$, sa profondeur de pénétration atteint $20 \mathrm{~m}$ dans le sable et environ $80 \mathrm{~m}$ dans les argiles. Les profondeurs sont exprimées en ms temps double, c'est à dire le temps que met l'onde acoustique pour 
faire l'aller/retour entre l'émetteur récepteur et l'interface sédimentaire. Pour en déduire une profondeur en mètres, il suffit de diviser par 2 cette échelle (pour n'avoir que le temps d'un seul trajet) puis de multiplier par 1600 m/s (jusqu'à 1800 m/s), temps de vitesse généralement estimé pour les sédiments marins superficiels. Cette vitesse peut être modifiée en fonction de la nature des sédiments.

Ainsi, cinquante-huit profils sismiques ont été acquis avec le boomer en mai 2012, au large des Pays de Monts, dont le plan de position est donné par la figure 1.
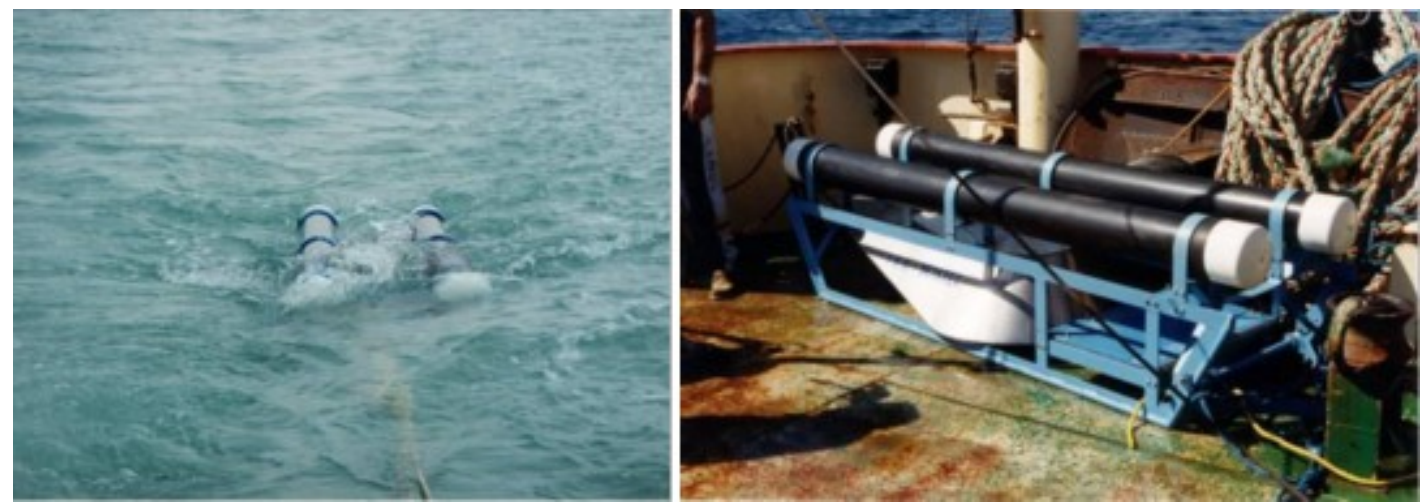

Figure 3. Le Boomer Seistec (sismique très haute résolution) - université de Caen.

\section{Résultats et discussion}

Six profils sismiques sur les cinquante-huit sont présentés sur la figure 4 (profils 8 et 9 , 10 et 11, 41 et 42). Ils révèlent différents environnements tels que de remplissages de paléo-vallées, quelques dépôts de sédiments plus ou moins grossiers (graviers, sables) et les affleurements des platiers rocheux.

Les profils 8 et 9, parallèles à la côte, entre St Gilles Croix de Vie et St Jean de Monts, montrent la succession de remplissages sédimentaires de paléo-vallées (relatifs à la remontée du niveau marin depuis 10000 ans BP (PINOT, 1980). Les différentes unités sont corrélables aux unités des affluents de la Loire sur le plateau continental, décrites par PROUST et al. (2010). Ces dépôts sont interrompus par l'affleurement de fragments du platier calcaire éocène, particulièrement visible sur les profils 9 et 11 . Le profil 10 montre un bel exemple de la couverture sableuse que l'on retrouve répartie le long de la structure géologique du Pont d'Yeu et, de façon plus imposante, au nord de l'Ile d'Yeu. Ce dépôt, appelé "la Sablière", présente une épaisseur de quelques mètres (3 à $4 \mathrm{~m}$ ) visible sur le profil 41 au large de la côte nord de l’Ile d’Yeu (figure $1 \&$ figure 4). 
XIII ${ }^{\text {èmes }}$ Journées Nationales Génie Côtier - Génie Civil

Dunkerque, 2-4 juillet 2014

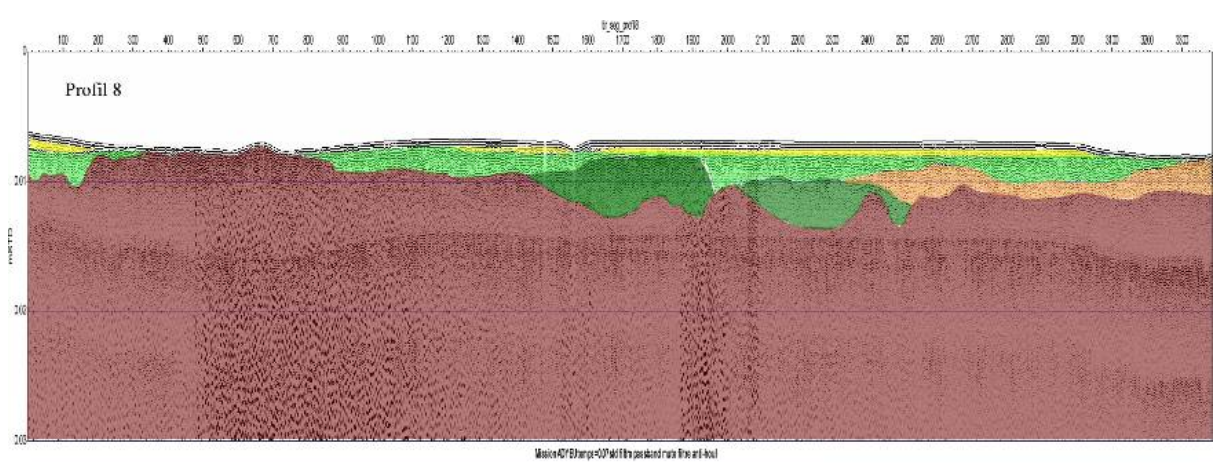

Whath')
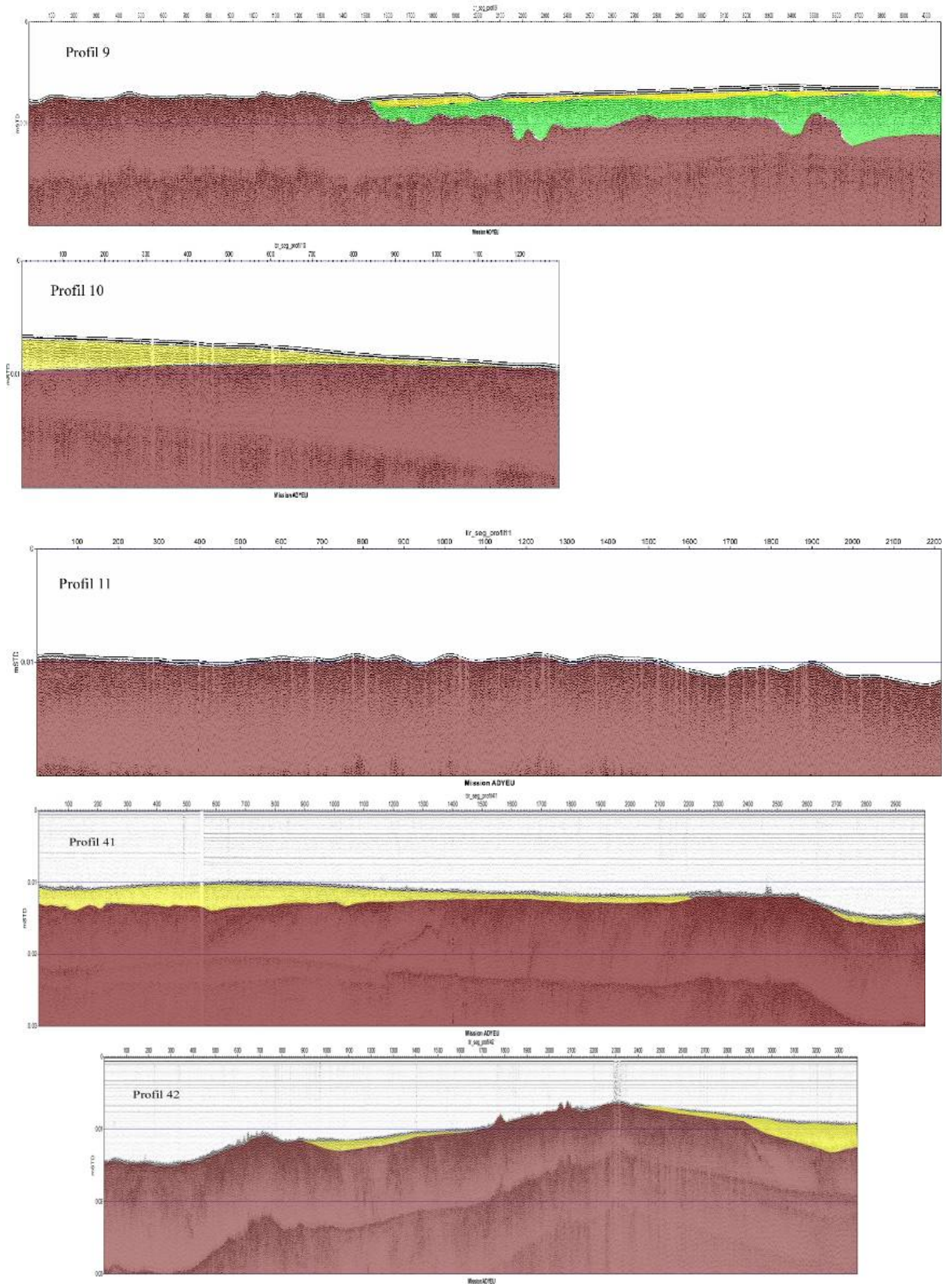

Figure 4. Différents profils acquis avec le Boomer Seistec. 
Ainsi les dépôts meubles et donc aisément mobilisables, semblent se répartir en trois zones principales : le long de la structure du Pont d'Yeu, sur la côte nord de l'Ile d'Yeu et le long du littoral vendéen, au pied du cordon dunaire. Cette répartition sédimentaire, visible sur les quelques profils a été comparée à la carte des formations sédimentaires réalisées par Vanney en 1977 (figure 5). Or la comparaison révèle que cette répartition n’a pas sensiblement changé depuis 40 ans. Une des explications les plus plausibles de cette "stabilité" sédimentaire pourrait être liée à la combinaison des deux facteurs suivants : une bathymétrie spécifique (l’Ile d’Yeu prolongée par le pont d’Yeu) et une houle d'ouest/sud-ouest dominante.

Les houles d'ouest, avec des hauteurs de $9 \mathrm{~m}$ régulièrement enregistrées, font de l'Ile d’Yeu, l'une des îles les plus exposées directement à l'action des houles avec Belle Île au nord-ouest.

Arrivant sur la côte sud rocheuse (falaises de gneiss et granite), cette houle va se réfracter de part et d'autre de l'Ile et va rabattre vers le pont d'Yeu les sédiments meubles. L'action d'érosion des houles sur les falaises sud est susceptible également d'alimenter directement "la Sablière" au nord. Notre hypothèse est que les dépôts sableux semblent rester piégés dans une cellule délimitée par l'Ile d'Yeu, le pont d'Yeu et le littoral vendéen. Ainsi même si l'érosion peut être importante sur les plages, en relation avec les tempêtes par exemple, le sable reste probablement stocké en domaine inter ou subtidal, mais ne sort pas du système.

Afin de vérifier cette hypothèse, l'échantillonnage des dépôts sableux, ainsi que l'identification de l'origine de ces sables est en cours.

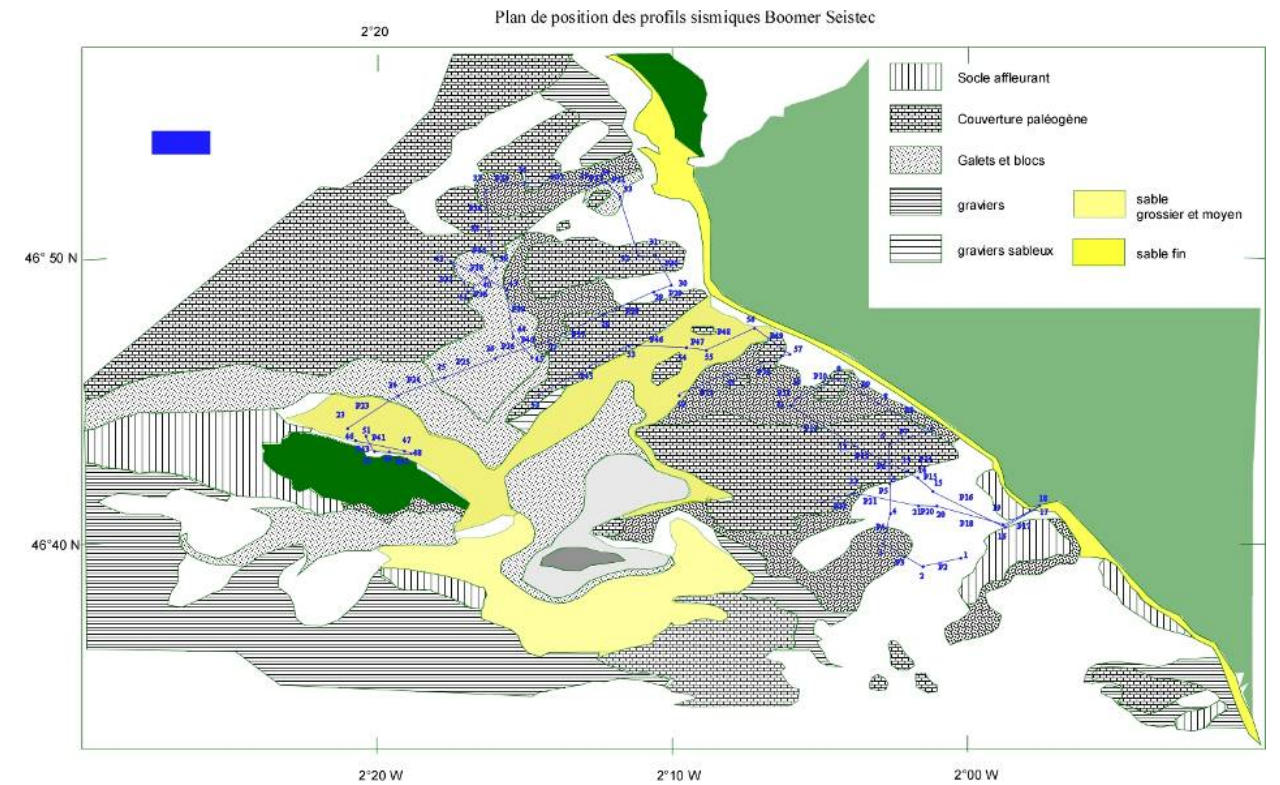

Figure 5. Le plan de position des profils sismiques réalisés (bleu) est superposé à la couverture sédimentaire du Coureau Ilais (VANNEY, 1977). 


\section{XIII ${ }^{\text {èmes }}$ Journées Nationales Génie Côtier - Génie Civil \\ Dunkerque, 2-4 juillet 2014}

\section{Conclusion}

Une mission de reconnaissance géophysique réalisée en 2013, révèle, en première approche, que la répartition des sédiments meubles sur la zone du Coureau Ilais semble quasiment identique à celle de 1977, décrite par Vanney. La stabilité de ces dépôts pourraient s'expliquer par l'action des houles dominantes qui se réfractent sur l'Ile d'Yeu et rabattent les sédiments sur les dépressions de la structure du Pont d'Yeu, le front nord de l'Ile d'Yeu et le long du cordon littoral vendéen. La cellule sédimentaire ainsi constituée confère une certaine stabilité au système, les dépôts restent piégés aux mêmes endroits, le reste étant balayé par les forts courants de marée et de houle. Une identification de la nature des sédiments prélevés devrait nous permettre dans une futur proche d'établir la provenance de ces sables.

\section{Références bibliographiques}

DHI (2007). Etude de connaissance des phénomènes d'érosion sur le littoral vendéen. Rapport DHI/GEOS, décembre 2007, 351 p.

PINOT (1980). Le Marais de Monts. Cahier Nantais n 18, pp 7-18.

PROUST J.N., RENAULT M., GUENNOC P., THINON I. (2010). Sedimentary architecture of the Loire River drowned valleys of the French Atlantic shelf. Bulletin de la Société Géologique de France, t 181, n², pp 129-149.

TERS M. (1961). La Vendée littorale, Etude de Géomorphologie. Thèse de doctorat d’État, Université de Rennes, 578 p.

VANNEY J.R. (1977). Géomorphologie de la marge continentale sud-armoricaine, SEDES, Publications de la Sorbonne, 473 p. 
Thème 3 - Instrumentation, mesures, imagerie et télédétection 